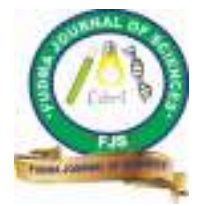

FUDMA Journal of Sciences (FJS)

ISSN online: $2616-1370$

ISSN print: 2645 - 2944

Vol. 4 No. 3, September, 2020, pp $43-47$

DOI: https://doi.org/10.33003/fjs-2020-0403-350

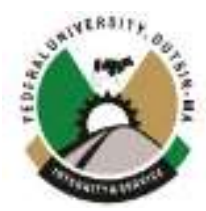

\title{
THE OCCURRENCE AND ANTIBIOGRAM OF Salmonella gallinarum ISOLATED FROM CLOACAL SWABS OF CHICKENS IN JOS SOUTH LOCAL GOVERNMENT AREA, PLATEAU STATE.
}

\author{
${ }^{1 *}$ Dasat, G. S. ${ }^{2}$ Danjuma, G. and ${ }^{3}$ Chuga A. P.
}

${ }^{1}$ Department of Food Science Technology, Department of Science, Plateau State Polytechnic, Barkin Ladi, Plateau State, Nigeria ${ }^{3}$ Department of Biochemistry, Veterinary Research Institute, Vom, Plateau State, Nigeria

*Corresponding authors' email and Telephone dsspoly@ yahoo.com. 08060885533

\begin{abstract}
Fowl typhoid caused by Salmonella gallinarum are controlled by biosecurity and vaccination by the poultry industry, however, these bacteria are still present in the poultry environment and outbreaks are often reported worldwide. Salmonella enterica serotype Gallinarum (S. gallinarum) is the causative agent of fowl typhoid (FT) in chickens causing heavy economic losses to poultry industry through mortality, reduced egg production and culling of breeding stocks. This study was designed to investigate the prevalence rate of Salmonella gallinarum from chicken cloacal swabs and antibacterial susceptibility in Jos South Local Government Area, using standard bacteriological methods. A prevalence rate of $4 \%$ was obtained out of the 100 samples. Serovar detected was Salmonella gallinarum4 (4 \%). Analysis of antibacterial susceptibility shows all isolates were $100 \%$ sensitive to Ciprofloxacin and Gentamycin and $100 \%$ resistant to Oxytetracycline, Erythromycin and Ampicillin. Fifty percent sensitive to Ceftriaxone while 1 (25\%) of the isolates were sensitive to Streptomycin and Amoxicillin-Clavulanic acid. Conclusively, this result indicates that Salmonella gallinarum can be isolated in cloacal swabs of asymptomatic broiler and layer chickens in the poultry farms. The use of drugs for prophylaxis and therapeutic purposes can be regulated and monitored to avoid transfer of resistant genes to human and other animals.
\end{abstract}

Keywords: Salmonella, Biosecurity, antibiotic, resistance, infection.

\section{INTRODUCTION}

Salmonella bacteria are gram negative facultative anaerobic rods from the family of Enterobacteriaceae (Yan et al., 2003). The Salmonella bacteria can catabolize a different carbohydrate into acid with the formation of gas and utilize citrate as the main source thereby producing $\mathrm{H}_{2} \mathrm{~S}$ and decarboxylate as by products. The bacteria convert lysine and ornithine to cadaverine and putrescine as well as grows in a temperature range of $35^{\circ} \mathrm{C}$ to $37^{\circ} \mathrm{C}$ (Barbara et al., 2000).

Shivaprashad, (2000) revealed that researchers in the veterinary sciences have identified Salmonella enterica sub spp enterica Serovar gallinarum as the main causative agent of fowl typhoid, and is placed into two distinct biovars namely, Gallinarum and Pullorum, which are designated as $S$. gallinarum and S. pullorum.

Agada et al (2014) listed septicaemic condition, large scale mortality, reduced egg production/quality, as well as poor hatched chicks due to infected eggs among poultry chickens as signs of fowl typhoid. Other indicators of fowl typhoid include anaemia, depression, difficulties in breathing and diarrhea causing adherence of faeces to the vent. Furthermore, poultry birds within the age bracket of 2-3 weeks are considered vulnerable and have the highest mortality to pullorum disease, but could be milder in older birds. Wray and Wray (2001) submitted that during breeding and laying periods, flocks could be easily exposed Salmonella infection which could result in low egg production and increased Trans-ovarian infection which is one of the major routes of transmission of diseases.
Jakirul et al. (2016) reported high mortality rate of poultry from some selected farms in Bangladesh reasons they attributed to Salmonella serovars infections after recovering the organism from cloacal swabs, and further stated that out of 44 positive cases of foul Salmonellosis $36.36 \%$ and $15.91 \%$ isolates were Salmonella gallinarum and Salmonella typhimurium respectively.

Fair and Tor (2014) submitted that antimicrobial resistance (AMR) is a situation whereby microorganisms impede the action of antimicrobial agents by developing mechanisms that mounts a wall of resistance against such actions. Consequently, the European Union (EU) introduced certain measures among which is prohibition of adding growth promoters in livestock feeds as a means of curtailing the rising cases of drug resistant among poultry birds.

Similarly, Agbaje et al. (2010) reported the outcome of an investigation on antimicrobial susceptibility test on five isolates of $S$. gallinarumin in a commercial poultry farm in Ogun State, Nigeria indicating a particular trend of resistance and susceptibility to some antibiotics including nalidixic acid, streptomycin and ciprofloxacin. Nevertheless, all were sensitive to Tetracycline, Neomycin, Ampicillin, Furazolidone, Ceftazidime, Sulphamethoxazole-Trimethoprim, Chloramphenicol, Amikacin, Amoxicillin-clavulanic acid, Gentamycin, Sulphonamide compounds, Cefotaxime and Apramycin. 
An instigation on antibacterial susceptibility tests carried out by Agada et al. (2014) in Jos among some poultry birds revealed a $100 \%$ resistance of Oxacillin against Salmonella serovars, $96.0 \%$ to Ampicillin, $93.9 \%$ to Tylosin, $83.7 \%$ to Ceftazidime, $69.4 \%$ to Ceftriaxone, $67.3 \%$ to Anicillin, $63.3 \%$ to Oxytetracycline and $55.1 \%$ to SulphamethoxazoleTrimethoprim.

Routinely, poultry farmers in Nigeria vaccinate their birds against the incidence of Salmonella gallinarum as part of control measures in the control of the spread of foul typhoid but despite these measures in place outbreaks still persist reasons attributed to resistant species of Salmonella gallinarum 9R strain (Fagbamila et al., 2017).

Agada et al. (2014) identified Pullorum disease (PD) and Fowl typhoid as the main bacterial diseases of poultry in Jos South accounting for heavy economic losses through mortality and reduced production therefore. These authors further suggest that adoption of adequate biosecurity measures and early vaccination are vital in the prevention of Salmonella infection in the poultry industry.

In recent years, Poultry farming business is on the increase in Plateau State particularly, Jos South Local Government area and with such expansion, any eventual outbreak of fowl typhoid is most likely to be very devastating and could spread to other parts of the State. The aim of this investigation therefore, is to determine the occurrence and antibiotic susceptibility of Salmonella gallinarum isolated from cloacal swabs of chickens in Jos South Local Government Area as part of control measures to minimize the spread of fowl typhoid within the study area and beyond.

\section{MATERIALS AND METHODS \\ Study area}

Jos South has its headquarters in the town of Bukuru at $9^{\circ} 48^{\prime} 00^{\prime}$ ' N, 8' 52'00" E, 1, $217 \mathrm{~m}$ above sea level and enjoys a more temperate climate. It has an area of $510 \mathrm{~km}^{2}$ located south of the state capital and a population of 311, 392 based on 2006 national census. It has an average temperature of 18 to $22^{\circ} \mathrm{C}$ and relative humidity of $60 \%$. The Local Government area is divided into four districts of Du, Gyel, Vwang and Kuru. It has an upland area with undulating hills, mountain outcrops, forest reserves, mining ponds, rivers, settlements, fertile Agricultural land for dry and rainy season farming. The main preoccupation of the people is both subsistence and commercial agricultural production with high proportion of the population involved in poultry farming.

\section{Sample size and sample collection}

In this study, one hundred Cloacal samples were collected from five randomly selected poultry farms in parts of Jos South Local Government Area designated as farms A, B, C, D \& E. Samples comprising of 20 each were collected aseptically directly from the cloaca of apparently healthy and diarrheic layer chickens using sterile swab from five different poultry farms as stated above. The swabs were transferred into labelled capped bottles containing $10 \mathrm{ml}$ of buffered peptone water (BPW) and transported to Microbiology section, Central Diagnostic Laboratory, National Veterinary Research Institute,
Vom in a thermos flask containing ice- packs for analysis within 24 hours.

\section{Detection method}

The processing was carried out according to International Standardization Procedure of the world orgaisation for animal health (OIE, 2015).

\section{Pre-Enrichment and Plating using Selective Media}

Pre- enrichment was carried out as detailed by Agada et al., (2014) and OIE, (2015). Briefly, samples were pre-enriched in $\mathrm{BPW}$ and incubated at $42^{\circ} \mathrm{C}$ for $24 \mathrm{~h}$. One $\mathrm{ml}$ of the preenrichment broth was transferred into tubes containing $9 \mathrm{ml}$ of Rappaport Vassiliadis Broth (RVB), incubated at $42^{\circ} \mathrm{C}$ for 24 h. A loopful of culture from RVB was sub cultured by streaking onto MacConkey agar (MCA) and Xylose Lysine Desoxycholate (XLD) media and incubated at $37^{\circ} \mathrm{C}$ for 24 hours.

\section{Preliminary Confirmation of Salmonella gallinarum}

After 24 hours incubation of plates at $37^{\circ} \mathrm{C}$, the presence of typical colonies of Salmonella based on cultural and morphological characteristics were examined and Presumptive isolates were Gram stained and purified onto MCA, XLD and NA.

\section{Biochemical characterization}

Classification of the isolates was carried out using different biochemical tests (triple sugar iron agar, citrate, urease, glucose, dulcitol, maltose, mannitol, sucrose, arabinose, xylose) as described by Agada et al. (2014) and OIE, (2015).

\section{Antibiotic susceptibility testing (AST)}

The antibiotic susceptibility testing was done using the KirbyBauer disk diffusion method (CLSI, 2018) using AmoxicillinClavulanic acid, Ciprofloxacin, Ceftriaxone, Oxytetracycline, Gentamycin, Ampicillin, Erythromycin and Streptomycin as the antibiotics of choice.

\section{Standardization of inoculum}

After 18-hour incubation period of the pure culture, sterile wire loop was used to pick 2 to 3 colonies of Salmonella isolate and emulsified in a tube to which $5 \mathrm{ml}$ of sterile physiological saline was previously added. The Salmonella isolate was standardized to $0.5 \mathrm{McF}$ arland Standard using a Nephalometer. Thereafter, $50 \mu \mathrm{L}$ of the broth was transferred into $5 \mathrm{ml}$ of Mueller-Hinton broth (Oxoid, UK) in a tube (CLSI, 2010).

\section{Inoculation of test plates}

After adjusting the turbidity of the inoculum suspension, a sterile cotton swab was dipped into the standardized suspension and inoculated by streaking unto the dried surface of plate containing 20ml Mueller-Hinton agar (Oxoid, UK).

\section{Application of dises on inoculated agar plates}

With the aid of disc dispenser, the antibiotic discs (Oxoid, UK) were evenly dispensed onto the surface of the inoculated agar plate and pressed down to ensure complete contact with the agar surface. The plates were inverted and incubated at $37^{\circ} \mathrm{C}$ for $24 \mathrm{~h}$. 
Examination and interpretation of AST results

Each plate was examined after $24 \mathrm{~h}$ of incubation and subsequently, diameters of the zones of complete inhibition were measured to the nearest whole millimeter, using a ruler and results interpreted based on the European Committee on Antimicrobial Susceptibility Testing (EUCAST) guidelines (EUCAST, 2019).

\section{RESULT}

The outcome from this investigation indicates that Salmonella gallinarum was isolated from 4 of the 100 cloacal samples examined, given a prevalence rate of $4.0 \%$ with Poultry farm $\mathrm{D}$ having the highest of 3 cases, followed by farm $\mathrm{E}$ with 1 case while Poultry farms A, B and C has no isolation as observed in Table 1).

Table1: Prevalence of Salmonella gallinarum in the five areas studied in Jos South LGA

\begin{tabular}{llll}
\hline Poultry farms & No. of Samples & No. Positive for & $\begin{array}{l}\text { \% Positive for } \\
\text { S. } \text { gallinarum }\end{array}$ \\
\hline A & & 0 & 0 \\
B & 20 & 0 & 0 \\
C & 20 & 0 & 0 \\
D & 20 & 3 & 15.0 \\
E & 20 & 1 & 5.0 \\
Total & 20 & $\mathbf{4}$ & $\mathbf{4 . 0}$ \\
\hline
\end{tabular}

Table 2: Antibiotic susceptibility profile of Salmonella gallinarum isolated from cloacal swab

\begin{tabular}{|c|c|c|c|c|}
\hline Antibiotic & $\mu \mathrm{g}$ & No. of isolates & Susceptible (\%) & Resistance (\%) \\
\hline Amoxicillin-Clavulanic acid & 30 & 4 & $1(25)$ & $3(75)$ \\
\hline Ciprofloxacin & 10 & 4 & $4(100)$ & $0(0)$ \\
\hline Ceftriaxone & 30 & 4 & $2(50)$ & $2(50)$ \\
\hline Oxytetracycline & 30 & 4 & 0 & $4(100)$ \\
\hline Gentamycin & 10 & 4 & $4(100)$ & $0(0)$ \\
\hline Ampicillin & 10 & 4 & $0(0)$ & $4(100)$ \\
\hline Erythromycin & 30 & 4 & $0(0)$ & $4(100)$ \\
\hline Streptomycin & 10 & 4 & $1(25)$ & $3(75)$ \\
\hline
\end{tabular}

Result of Table 2 indicates the antibiotic susceptibility profile of various antibiotics used on the Salmonella gallinarum isolated. The results showed $100 \%$ resistant to three brands of antibiotics namely Oxytetracycline, Ampicillin and Erythromycin. However, Ciprofloxacin and Gentamycin were sensitive to all the isolates representing $100 \%(\mathrm{P}>0.05)$.

\section{DISCUSSION}

Results obtained from this investigation reveals a generally low infection rate of $S$. gallinarum in the study areas where only 4 out of the 100 samples examined were positive which is statistically insignificant $(\mathrm{p}>0.05)$ as observed in Table 1 . This low incidence could be attributed to adequate biosecurity measures put in place and proper vaccination of chickens within the poultry farms. The isolation rate of $S$. gallinarum obtained in this study was however higher compared with the result of Jones (2001), who reported a $0(0 \%)$ isolation from Cloacal swabs of local chickens in Poland.

This result is in agreement with the work of Garba et al. (2010), whom reported a $4 \%$ isolation rate out of 150 cloacal swabs sampled from broiler chickens, suggesting a low incidence of S. gallinarum. In contrast, Yhiler and Bassey (2015), reported a high prevalence of $59.1 \%$ Salmonella species from cloacal swabs of poultry birds in Calabar, Cross River State, Nigeria.

Increasing rate of antibiotics resistance in poultry birds is well documented (Winokur et al., 2000. Conseqeuntly, this investigation reveals that $S$. gallinarum isolated from cloacal swabs were significantly ( $p>0.05$ ) resistant to Ampicillin, Oxytetracycline and Erythromycin, representing $100 \%$ resistance, followed by Nalidixic acid with $75 \%$ resistance (Table 2).

Ciprofloxacin was found to be effective against all Salmonella isolates representing $100 \%$ susceptibility as observed in Table 2. However, the relationship between $S$. gallinarum and antibacterial drugs tested was not statistically significant $(p>$ $0.05)$. This work is in agreement with the findings of Clin and Diagn (2013).

The significant resistance observed for Ampicillin, Oxytetracycline and Erythromycin in this study (Table 3) could be as a result of improper drug administration or the use of expired drugs in the study area which is possibly the most important factor encouraging the emergence of resistant species of Salmonella (Agada et al., 2014). Consequently, Okoli et al. (2006) reported that antibacterial susceptibility of Salmonella isolates is usually dynamic and varies with time and environment. This therefore, requires the periodic screening of Salmonella for their antibacterial drug susceptibility profile in the study area as well as different local government areas of Plateau State.

It is therefore important to note that the indiscriminate use of antibacterial drugs without proper regulation and right 
administration could encourage the emergence of resistant Salmonellae strains.

Recent reports (Agada et al. (2014) identified wrong drug administration in the field of veterinary medicine as responsible for increasing cases of bacterial strains resistance, therefore multiple antibacterial drug resistance could be transferred through conjugation from resistant strains of $S$. gallinarum to another serovar by means of plasmid. Therefore, the high resistance of $S$. gallinarum isolated in this study to Oxytetracycline, which is a common drug used by poultry farmers for prophylaxis and treatment calls for caution (Kamelia et al., 2019).

The $100 \%$ resistance recorded in this study to Oxytetracycline is higher than the reports documented in Senegal $46 \%$ (BadaAlambedji et al., 2006) and $36 \%$ in Portugal (Antunes et al., 2003).

Oxytetracycline antibiotic has been a drug of choice for ages commonly administered to poultry birds ranging from day-old chicks to broiler chickens. Therefore, Jones, (2001) posited that early exposure of animals to such drugs as well as prolong exposure could result in the birds developing resistance to the antibiotic as they grow to maturity.

High level of Ampicillin resistance $(100 \%)$ observed in all the isolates was in agreement with Suresh et al (2006) and Agada et al. (2014). The low level of susceptibility observed in cephalosporin, a major antibacterial drug used in treating salmonellosis, shows that the drug could be compromised as reported by Yhiler et al. (2015). In Nigeria, Salmonella serotypes with reduced macrolides and cephalosporin resistance from human and poultry has been documented (Agada et al., 2014).

In this study, Ciprofloxacin and Gentamycin showed high leve of susceptibility $(100 \%)$ to all the Salmonella isolates. Ifeanyi et al. (2013) conducted a similar study and reported an increased level of susceptibility to Ciprofloxacin and Gentamycin which is in agreement with the present study. Nevertheless, Enabulele et al. (2010) reported high resistance of Salmonella isolates to Gentamycin and Ciprofloxacin.

\section{CONCLUSION}

This study recorded low incidence of fowl typhoid due to Salmonella gallinarum infection among broiler and layer chickens but recorded high resistance to commonly used antibiotics (Ampicillin, Oxytetracycline and Erythromycin) in parts of Jos South Local Government Area of Plateau State. This, therefore, is an indication that poultry farmers within the study area possibly adopted adequate biosecurity measures The high resistance to the mentioned antibiotics calls for the need to educate Poultry farmers on the danger of indiscriminate use of antibiotics and medication as these drugs can pass on through the food chain to humans. Poultry farmers should also be encouraged to practice rapid and regular vaccination of chickens against Salmonella infection.

\section{REFERENCES}

Agada, G. O. A., Abdullahi, I. O., Aminu, M., Odugbo, M., Chollom, S.C., Okeke, L.A. \& Okwori, A. E. J. (2014). Risk factors Associated with Salmonella species Contamination of Commercial Poultry Farms in Jos, Plateau State, Nigeria. International Journal of Current Research, 6 (4), 6292-6301.

Abase, M., Davies, S., Oyekunle, M. A., Ojo, O.E., Fasina, F.O. \& Akinduti, P.A. (2010).

Observation on the Occurrence and Transmission Pattern of Salmonella gallinarum in Commercial Poultry in Ogun State, South Western Nigeria. African Journal of Microbiology Research, 4(9): 796-800.

Antunes, P., Réu, C., Carlos, J. S., Peixe, L.\& Pestana, N. (2003). Incidence of Salmonella from Poultry Products and their Susceptibility to Antimicrobial Agents. International Journal of Food Microbiology, 82(2), 97-103

Bada-Alambedji, A., Fofana, M., Seydi, A. \& Akakpo, J. (2006). Antimicrobial resistance of Salmonella isolated from poultry carcasses in Dakar (Senegal). Braz J Microbiol, 37 $510-515$

Barbara, M. L., Baird-Parker, T.C., \& Grahame, W.G. (2000). The Microbiological Safety and Quality of Food (II). Gaithersburg, Maryland, USA: Aspen Publishers Inc. p.1234.

Clin, J. \& Diagn, R. (2013). Revised Ciprofloxacin Breakpoints for Salmonella: Is it Time to Write an Obituary? Journal of clinical and diagnostic research. 7(11): 2467-2469. doi: $10.7860 / J C D R / 2013 / 7312.3581$

Clinical and Laboratory Standards Institute (CLSI). (2018). Performance Standards for Antimicrobial Susceptibility Testing. M100, $28^{\text {th }}$ ed. Wayne (PA), Wayne (PA): the Institue.

Enabulele, S. A., Peace, O. Amune, O. A. \& Wakili, T. A. (2010). Antibiograms of Salmonella Isolates from Poultry Farms in Ovia North East local government area, Edo State, Nigeria. Agric. Biol. J. N. Am. 1(6): 1287-1290

The European Committee on Antimicrobial Susceptibility Testing (EUCAST). (2019). Definitions of clinical breakpoints and epidemiological cut off values. Modified definitions valid from 1 January, 2019.

Fair, R. J. \& Tor Y. (2014). Antibiotics and Bacterial Resistance in the 21st Century. Perspect. Med. Chem. 6:14459. doi: 10.4137/PMC.S14459.

Fagbamila I. O; Barco L; Mancin M; Kwaga J; Ngulukun S.S; Zavagnin P; Lettini, A. A., Lorenzetto, M; Abdu P. A; Kabir, J., Umoh, J., Ricci, A. \& Muhamad, M. (2017).

Salmonella serovars and their Distribution in Nigerian Commercial Chicken layer Farms. PLoS ONE 12(3): e0173097.

Garba, A., Bolajoko, B. M., Barde, A. Ahmed, I., Sa'adatu, I. Agang, A. S., Abdullahi, H. A., Bakari, U. I. J., Uraki, A. T., Abdurrahman, A. \& Goji, J. N. (2010). The Threat of Salmonellosis to Commercial Poultry Production in Adamawa 
state, Nigeria. Sokoto Journal of Veterinary Sciences, 8(1\&2): 50-52. http://dx.doi.org/10.1007/s11250-014-0680-8

Ifeanyi, C. I.C., Bassey, E. B., Ikeneche, N. F., Isu, R. N. \& Akpa, A. C. (2013). Prevalence and Antimicrobial Susceptibility of Salmonella Specie Associated with Childhood Acute Gastroenteritis in Federal Capital Territory Abuja, Nigeria. British Microbiology Research Journal, 3: 22310886.

Jakirul, M.D. I., Mahbub-E-Elahi1, A.T.M., Ahmed1, T., Md. Kamrul, M. D. H. \& Jones, B.D. (2016): Isolation and Identification of Salmonella spp. from Broiler and their Antibiogram study in Sylhet, Bangladesh. Journal of Applied Biology \& Biotechnology,4 (03), 046-051. DOI: 10.7324/JABB.2016.40308.

Jones, M.A., Wigley, P., Page, K.L., Hulme, S.D. and Barrow, P.A. (2001). Salmonella enterica serovar Gallinarum requires the Salmonella Pathogenicity Island 2 type III Secretion System but not the Salmonella Pathogenicity island 1 type III secretion System for Virulence in Chickens. Infection and Immunity, 69: 5471-5476.

Kamelia, M., Osman, J. B., Ahmed, O., Ayman, E., Aalaa., S., Mai, D.S. I. Mai. \& H. H. (2019). Poultry as a Vector for Emerging Multidrug Resistant Enterococcus spp.: First report of Vancomycin (van) and the Chloramphenicol-florfenicol (cat-fex-cfr) Resistance Genes from Pigeon and Duck faeces. Microbial Pathogenesis. 128, 195-205.

Okoli, I. C., G. E. Endujihe, \& I. P. Ogbuewu. (2006). Frequency of Isolation of Salmonella from Commercial Poultry Feeds and their Antimicrobial Resistance Profiles, Imo State, Nigeria. Journal of Health and Allied Science,5 (2): 3.
Shivaprashad, H. I. (2000). Fowl Typhoid and Pullorum Disease. Review of Science Technology, 19 (2): 405-24.

Suresh, T., A. A. M. Hatha, A. A. M., Sreenivasan, D., N. Sangeetha, N. \& Lashmanaperumalsamy. P (2006). Prevalence and Antimicrobial Resistance of Salmonella enteritidis and other Salmonellas in the Eggs and Egg-storing trays from Retails Markets of Coimbatore, South India. Food Microbiology, 23: 294-299.

Winokur, P. L A., Brueggemann, D. L., DeSalvo, L., Hoffmann, M. D., Apley, E. K., Uhlenhopp, M. A., \& Pfaller, G. V. (2000). Doern.Animal and Human Multidrug- Resistant,

Cephalosporin resistant Salmonella Isolates Expressing a Plasmid Mediated CMY-2 AmpC beta-lactamase. Antimicrobial Agents of Chemotherapy 44: 2777-2783.

World Organization for Animal Health (OIE) (2015). Animal report use of antimicrobial agents in animals-better understanding of the global situation: OIE: Paris, France.

Wray, C. \& Wray, A. (2001): Salmonella in Domestic Animals, book review. Journal of Veterinary Microbiology 81, 281-282. Yan, S. S., Pandrak, M. L., Abela-Rider, B., Punderson, J.W., Fedorko, D. \& Foley, S. L. (2003). An overview of Salmonella typing public health perspectives. Clinical and Applied Immunology Reviews 4, 189-204.

Yhiler, N.Y. \& Bassey, B. E. (2015). Antimicrobial Susceptibility Patterns of Salmonella Species from Sources in Poultry Production Settings in Calabar, Cross River State, Nigeria. American Journal of Health Research. 3, (2) 7681.doi: 10.11648 